\title{
Issues in Quantitative Modelling in the Early Warning of Refugee Migration
}

\author{
Susanne Schmeidl and J. Craig Jenkins
}

\begin{abstract}
This article discusses the problems associated with indicator analysis for the purpose of early warning. While the authors endorse the idea of quantitative EWM, they are sceptical of the understanding that many have of what EW analysis entails. In this article, they identify the limits of quantitative EW analysis and address many of the major problems that confront those who are committed to quantitative EW analysis. In particular, the authors discuss the following issues that need to be addressed when engaging in quantitative early warning analysis: the problem of "late warning;" problems of contextual sensitivity; problems of temporal development; data availability and measurement; and problems with the definition of the appropriate unit of analysis.
\end{abstract}

\section{Résumé}

Cet article traite des problèmes rattachés d̀ l'utilisation d'indicateurs en matière d'alerte préventive. Tout en faisant état de la pertinence de l'élaboration de modèle quantitatifs, les auteurs émettent des doutes d propos des conclusions obtenues à partir de ces analyses. Les limites de ces modèles et les principaux obstacles rencontrés dans la conduite de ces analyses sont identifiés. Les problèmes rattachés à une alerte lancée en retard, au caractère évolutif des situations conflictuelles, à la disponibilité et a l'éroaluationde l'information, d̀ la définition et au choix de la méthode

Dr. Susanne Schmiedl, Coordinator, Prevention/ Early Warning Unit, Centre for Refugee Studies, York University.

J. Craig Jenkins, Professor of Sociology; Faculty Associate, Mershon Center for International Security; and Director of Comparative Social Change Program, Ohio State University.

An earlier version of this paper was presented at the International Studies Association meeting, San Diego California, April 18, 1996. d'analyse appropriée ainsi qu'a l'article et da la susceptibilité des parties impliquées sont évoqués de manière particulière.

Early Warning Models (EWMs) have largely been used with success in the forecasting of ecological disasters such as droughts or storms but are as yet unproven in the forecasting of humanitarian disasters such as refugee migrations, human rights violations, and conflict. Recently, a number of scholars have begun systematic work on the development of quantitative EWMs so that eventually we will be able to foreshadow humanitarian disasters and thereby inform both policymakers and the academic community of the risks of such events. For example, there are EWMs of communal conflicts (Gurr 1994), genocides (Fein 1993), politicides (Harff 1994), armed conflicts (Bond and Vogele 1995) and population movements (Clark 1983, 1989). Each of these EWMs suggests that eventually we will be able to deal with humanitarian disasters in a fashion similar to ecological ones. Once early warning signs are identified, such information can be "received, digested and brought into decision-making by those who can prevent a man-made disaster or cope with its results" (Gordenker 1986, 185).

While we endorse the idea of quantitative EWM, we are skeptical of the understanding that many have of what EW analysis entails. In this paper, we identify the limits of quantitative EW analysis and address many of the major problems that confront those who are as committed as we are to quantitative EW analysis.

Our main point can be best demonstrated through a comparison with ecological EWMs. Humanitarian EW analysis is inherently reactive. With ecological EWMs, once we know that a storm, flood or famine will occur, we can only prepare to lessen its consequences by properly sheltering or evacuating people and providing relief. In the case of humanitarian disasters, however, we have two options: We can try to provide relief, or, optimally, we can try to prevent the disaster from occurring. Since human disasters have an intrinsic element of human agency, it is always possible to negotiate or apply pressure for peaceful (re)solutions. This, of course, makes humanitarian EWMs all the more appealing since they might allow for the possibility of preventive intervention. Yet, this very aspect of human agency also makes the early warning of humanitarian disasters much more unpredictable than the early warning of ecological disasters. In fact, we would argue that this makes the goals of humanitarian EWMs qualitatively different. Instead of attempting to develop predictive models, our primary aim should be to foreshadow humanitarian disasters and subsequently inform human actors about potential sources of humanitarian disaster. Because of this inherent reactivity of human action, the major aim of early warning is preventive, rather than being strictly a forecasting device. As such, humanitarian early warning requires a detailed analysis of three important elements: actors, situations, and contexts. Although we do not believe that EWMs will ever to be able to predict the exact timing of the outbreak of a humanitarian disaster, we do believe that they could eventually foreshadow such events and thereby alert the policymakers and the international community to conditions in countries which are likely to lead to a crisis. Therefore, the main issue is not to predict exactly when and where a humanitarian disaster will occur, but to learn as much as possible about the 
underlying patterns of such crises in the past (which includes quantitative analysis) and integrate such information into a context rich study of a specific region in crisis, which, in turn, should be derived from extensive casebased knowledge. Such knowledge can then be used by policymakers to develop scenarios and strategic alternative responses to prevent, or inhibit, the escalation of the factors most likely to cause conflict and refugee migration.

In the past, then, early warning has been interpreted as necessitating a model that adequately predicts humanitarian disasters. As social scientists, we agree with this goal for scientific purposes. However, for EWM purposes, this is potentially misleading. It is important to understand the limits of EWMs with the respect to humanitarian disasters at the outset and to address problems of indicator construction within that context.

\section{Past EWM Research}

Currently, there are numerous efforts by academics and policymakers to develop early warning on quantitative basis. As previously mentioned, in the past such academic efforts have largely and the UN Department of Humanitarian Affairs (DHA). Although partially engaging in case studies, these works essentially attempt to construct valid indicators that can be used with large samples of countries and regions to predict specific types of humanitarian disasters. However, we think such work (including our own) has to address a number of problems:

1. the problem of "late warning"

2. problems of contextual sensitivity

3. problems of temporal development

4. data availability and measurement, and

5. problems with the definition of the appropriate unit of analysis.

\section{The Problem of "Late Warning"}

"Late warning" is a major problem, particularly for academic researchers, due to a reliance on indicators that are collected from official documents (including event data derived from electronic newswires), or indicators that lag one or more years behind the occurrence of relevant events. This time lag impairs a timely analysis and leads to a "late warning": an analysis that shows whether or not we could have predicted a certain event or pattern of such events. While "late warning" (or

\section{Instead of attempting to develop predictive models, our primary aim should be to foreshadow humanitarian disasters and subsequently inform human actors about potential sources of humanitarian disaster.}

relied on a formal model approach, in search of the best indicators. Notable efforts include the monitoring of major armed conflicts (Singer 1994; Wallensteen and Sollenberg 1995; Bond and Vogele 1995), ethnic conflict (Gurr 1993), genocide (Fein 1993), politicide (Harff 1994), refugee migrations (Schmeidl 1995), environmental disasters (Homer-Dixon 1994; Lee 1994), and human rights (Jongman 1994). Among policymakers, the most prominent recent examples are the State Failure Project (Gurr 1995), sponsored by the United States government, and the efforts by the United Nations High Commissioner for Refugees (UNHCR) in scientific terms, retrodiction) is a very useful exercise in testing certain indicators, it does not really fit the aim of EWM, that is early warning. The computerization of information and the ability to code from computer generated news has improved this considerably, and we suspect that in real time forecasting may soon be feasible given the automation of event data collection. Nonetheless, this remains a major problem.

Even assuming automation reducess the time lag inherent in "late warning," significant problems remain. First, areas in crisis are typically underreported in the standard interna- tional wire services that we all depend on for information. Journalists and governmental experts typically arrive on the scene after the events have become troublesome. Second, relevant information may be withheld from widespread distribution (such as on the Internet) and thus remain inaccessible. Third, the existing automated systems are unable to contextualize critical information and thus make it relevant to policymakers. For example, in the PANDA system (with which one of the authors is associated), we may be able to accurately count the number and characteristics of wide-spread political protests, but we cannot identify the goals or specific meanings of these events, and thus we are unable tojudge the extent to which they are likely to lead to a crisis. At this point, we need to integrate quantitative indicators with the expertise found in each country, thereby bringing expert methods together with standard indicator approaches.

\section{Contextual Sensitivity}

Learning of political events out of context leads to the second problem, contextual sensitivity. In the early warning literature there is an acknowledgement of the multi-leveled conditions that can produce humanitarian disasters. These are usually distinguished as root (long-term) causes, proximate (medium-term) events, accelerating factors, triggering events, and intervening conditions. Typically these various conditions are treated as if they were mutually exclusive, but in fact, we suspect they are not. Clark (1989), for example, argues that in the early warning of refugee disasters, proximate events can also be intervening factors. Collective action among the population, for instance, could either a) lead to refugee out-migration through a threat to the government, which, in turn increases repression, or b) present an alternative to flight. The outcome of collective action depends on context, especially the availability of escape routes, the responsiveness of the regime, and so on. In quantitative modelling context sensitive measures 
that capture this complexity must be devised. We recommend strongly that EW analysts begin considering methods of contextual analysis widely used in the social sciences and adapt these to their purposes. This also points to the need to become knowledgeable about specific countries and cases, so that we can better understand how these complex contexts work.

\section{The Timing Problem}

A third problem is the timing of indicators. We may never resolve the issue of exact timing, since "each incident of forced migration has particular characteristics" (Gordenker 1992, 4). Long-term (or root) causes may occur years or even decades before the exodus, while medium-term (or proximate) causes may occur only months still very little guidance in the literature as to how long it really takes for certain events to lead to humanitarian disasters. While it might be self-evident that genocide and war will lead to refugee migration, the temporal structure of these developments can vary. In some cases, the outbreak of a war and/or genocide may directly correspond with the start of refugee movements; then again, it may take months, or longer for people to pick up and leave. Migratory movements also vary in accordance with different types of generalized violence. During civil wars, people have been known to refuse to leave despite intensive violence (e.g. Peru); while elsewhere people turn to resistance and/or flight readily. What this suggests is that we need to experiment extensively with

\section{The main problem with the unit of analysis is the uneven coverage of countries and regions, especially with the creation of new states and the increase in subnational or regional conflicts and disasters.}

before out-migration. Since the time point of the causes could be decades before the refugee movement occurs, it may be difficult to find indicators that fit into a model aimed at explaining refugee migration.

Triggering events are the most difficult to place. Theoretically, they would occur almost simultaneously with, or only days before, flight. In addition, most conventional methods in the social sciences (including time-series analysis) are unable to evaluate the close timing associated with triggering events. Due to these problems, EWM researchers have shied away from more immediate causes, such as triggering events, and typically focused on root, proximate and accelerator factors. However, it is important to point out that for policy purposes, triggering events are critical in preparing for emergency relief.

In our own research on refugee early warning, we have experimented with several time lags, up to ten to fifteen years for some root causes. Yet there is varying time-lags and to incorporate an awareness of contextual variation into this temporal process.

\section{Availability of Data}

The largest problem of all is suitable and reliable data. Most of us are intensively involved in indicator construction precisely because of the absence of suitable and reliable indicators of relevant processes. Such indicators need to provide both geographic and temporal coverage; otherwise we will not be able to generate useful assessments. However, important variables such as income inequality or land inequality are only available for a small number of countries and for a limited time period. Furthermore, sometimes regional information might be very important, which is even harder to find (we will discuss this 'unit of analysis' problem later). Thus, a researcher engaged in quantitative analysis is often compelled to ignore important factors (e.g., inequality) or important cases (e.g., the poorest countries) because of lack of information. Related to this is the problem of access (including security and proprietary data). Government agencies and transnational corporations often have relevant data, but are unwilling to share such sensitive information.

\section{Measurement Issues}

We often have to rely on very crude indicators for important events. For example, we may know whether or not there was a war or a genocide, but it may be more important to know the intensity of such a war or genocide when trying to predict a certain outcome such as refugee migration. Death estimates of such humanitarian disasters, however, are problematic. After all, mass graves in Bosnia and Rwanda are just now telling the tale of the extent of genocides that took place few years ago and that have been a subject of dispute among several sources. Similarly, we may never know the exact number of people affected in a war. This information, often very crucial, is almost impossible to obtain. We do not know the number of deaths (let alone the toll of the injured), of houses burned or destroyed, of women raped. All this could aid us in assessing the intensity of a humanitarian crises, yet the information is unavailable, and we are forced to rely on very crude estimates. This discussion can be extended to the amount of weaponry involved in a war or dispute, the number of people participating in conflict and protest, etc. All this information is often very sensitive and thus not readily available; when available, its veracity is highly contested.

These measurement problems greatly limit the type of EWMs that can be constructed. Schmeidl's (1995) analysis of the early warning of refugee migration illustrates this issue. Although she was able to predict refugee migration over a twenty year period (1971-1990) across 109 countries, she encountered several problems. First, there were many countries that did not follow the general pattern described in the model. Some countries expelled considerably more refugees 
than expected and some countries less. This suggests that the major indicators were not sensitive enough to explain refugee migration. However, crude estimates of the intensity of wars and genocides (instead of a simple dichotomous variable of occurrence and non-occurrence) did not improve the results and, in some cases, produced inferior results. This may reflect poor quality estimates of the intensity of violence, but it may also reflect the difficulty of linking violence to particular time periods. Thus, a simple dichotomous variable proved statistically more useful, despite the fact that substantively it should be inferior.

\section{The Unit of Analysis}

The main problem with the unit of analysis is the uneven coverage of countries and regions, especially with the creation of new states and the increase in subnational or regional conflicts and disasters. The breakup of the former Soviet Union and Yugoslavia, and Ethiopia, along with the growth of regional conflicts, have created a need for a new level of analysis for which we are lacking (for the most part) suitable data. Cross-national scholars have focused on developing national indicators but largely ignored sub-national differences. We are all aware of countries with major regional or internal inequalities and differences but, aside from crude estimates (e.g., sectoral inequality, ethnic differences) we have neglected these indicators. For the policymakers, however, such sub-national measurement is becoming of increasing importance.

Similarly, the most important indicators are often relational indicators, such as the relationships between different groups and populations, yet these are barely explored. Gurr (1993) and associates have made an heroic effort to tackle this issue with ethnic minorities. This kind of research needs to be extended to other kinds of vulnerable populations (e.g., women, children, the elderly, regional subgroups) so that we can accurately gauge the populations at risk.

\section{Conclusion}

Despite our scepticism, our aim has not been to disavow the importance or the eventual promise of quantitative EWM. In fact, we are practitioners of the art as well as its champions. Our aim has been to identify the major analytic problems that quantitative EW assessment currently confronts so as to promote a better understanding of the task ahead. In an ideal world, EWM will eventually be able offer: 1) a global reach in terms of the number of countries and time periods concerned;2) a comparison of positive as well as negative cases of the development of humanitarian disasters and their major determinants; and 3) the incorporation of sub-national and regional information as well as relevant indicators of the risks of specific populations. Eventually, we will have a deeper understanding of the causes of specific types of humanitarian disasters, their timing, and their contextual sources. Ultimately, we will be able to use the reactivity of human agency to our advantage by providing timely information that can be used for policy purposes as well as academic analysis.

\section{References}

Aga Khan, Sadruddin. 1981. "Study on Human Rights and Massive Exodus: Question of the Violation of Human Rights and Fundamental Freedoms in any Part of the World, with Particular Reference to Colonial and Other Dependent Countries and Territories." Special Report to the Commission on Human Rights, 38th Session, United Nations Economic and Social Council GE, 82-10252.

Bond, Douglas, and Bill Vogele. 1995. "Profiles of International Hotspots." Centre for International Affairs, Harvard University.

Clark, Lance 1983. Early Warning of Refugee Mass Influx Emergencies. Washington, D.C.: Refugee Policy Group.

- 1989. Early Warning of Refugee Flows. Washington, D.C.: Refugee Policy Group.

Dedring, Jürgen. 1994. "Early Warning and the United Nations." The Journal of EthnoDevelopment 4(1):98-105.

Fein, Helen. 1993. "Accounting for Genocide after 1945: Theories and some Findings." International Journal on Group Rights 1:79106.
-1994. "Tools of Alarms: Uses of Models for Explanation and Anticipation." The Journal of Ethno-Development 4(1):31-36.

Gordenker, Leon. 1986. "Early Warning of Disastrous Population Movement." International Migration Review 20(2):170-93.

_- 1992. "Early Warning: Conceptual and Practical Issues." In Early Warning and Conflict Resolution, edited by Kumar Rupesinghe and Michiko Kuroda, 1-15. London, England: The MacMillan Press, Ltd.

Gurr, Ted Robert. 1993. Minorities at Risk: $A$ Global View of Ethnopolitical Conflict. Washington, D.C.: United States Institute of Peace Press.

- 1994. "Testing and Using a Model of Communal Conflict for Early Warning." The Journal of Ethno-Deoelopment 4(1):20-25.

— 1995. "The State Failure Project: Early Warning Research for International Policy Planning." Paper presented at the Annual Meetings of the International Studies Association, Chicago, IL.

Harff, Barbara. 1994. "A Theoretical Model of Genocide and Politicides." The Journal of Ethno-Development 4(1):25-31.

Homer-Dixon, Thomas. 1994. "Environmental Scarcities and Violent Conflict: Evidence from Cases." International Security 19(1):5-140.

Jongman, Albert. J. 1994. "The PIOOM Program on Monitoring and Early Warning of Humanitarian Crises." The Journal of Ethno-Development 4(1):65-72.

Lee, Shin-wha. 1994. "Environmental Change, Refugees, and Conflict in the Third World: A Framework for Inquiry Applied to Case Studies of Sudan and Bangladesh." Ph.D. Dissertation, Political Science, University of Maryland, College Park.

Ramcharan, B. G. 1991. The International Law and Practice of Early-Warning and Preventive Diplomacy: The Emerging Global Watch. The Hague: Martin Nijhoff Publishers.

Schmeidl, Susanne. 1995. "From Root Cause Assessment to Preventive Diplomacy: Possibilities and Limitations of the Early Warning of Forced Migration." Ph.D. Dissertation, Sociology, The Ohio State University.

Singer, J. David. 1994. Correlates of War Project: Data Files. The University of Michigan.

Wallensteen, Peter, and Margareta Sollenberg. 1995. "After the Cold War: Emerging Patterns of Armed Conflict 1989-94." In States in Armed Conflict 1994, edited by Margareta Sollenberg, 7-23. Report No. 39, Department of Peace and Conflict Research, Uppsala University. $\square$

(C) Susanne Schmeidl and J. Craig Jenkins, 1996. This open-access work is licensed under a Creative Commons Attribution-NonCommercial 4.0 International License, which permits use, reproduction and distribution in any medium for non-commercial purposes, provided the original author(s) are credited and the original publication in Refuge: Canada's Journal on Refugees is cited. 